\title{
Association Between Interleukin-12 and Sarcopenia
}

\author{
Yuan-Yuei Chen ${ }^{1-3}$ \\ Tung-Wei $\mathrm{Kao}^{3}$ \\ Yi-Lin Chiu (1D ${ }^{4}$ \\ Tao-Chun Peng ${ }^{3}$ \\ Hui-Fang Yang $\mathbb{D}^{3}$ \\ Wei-Liang Chen (D) $^{3,4}$ \\ 'Department of Pathology, Tri-Service \\ General Hospital, School of Medicine, \\ National Defense Medical Center, Taipei, \\ Taiwan, Republic of China; ${ }^{2}$ Department \\ of Pathology, Tri-Service General \\ Hospital Songshan Branch, School of \\ Medicine, National Defense Medical \\ Center, Taipei, Taiwan, Republic of China; \\ ${ }^{3}$ Division of Geriatric Medicine, \\ Department of Family and Community \\ Medicine, Tri-Service General Hospital, \\ School of Medicine, National Defense \\ Medical Center, Taipei, Taiwan, Republic \\ of China; ${ }^{4}$ Department of Biochemistry, \\ National Defense Medical Center, \\ Taiwan, Republic of China
}

Background: Emerging studies have proposed that cytokines secreted following macrophage polarization may contribute to skeletal muscle aging. The current study primarily aimed to determine whether these cytokines have an impact on the progression of sarcopenia in an elderly population.

Methods: In total, 120 elderly adults aged 65 years and older who underwent health examinations from 2015 to 2019 were included in this retrospective study. Sarcopenia was based on the criteria proposed by the European Working Group on Sarcopenia in Older People in 2019. Macrophages and cytokines datasets were obtained from Gene Expression Omnibus (GEO) database. Comprehensive assessments were performed for muscle strength, muscle mass, gait speed, tumor growth factor- $\beta$ (TGF- $\beta$ ), and interleukin-12 (IL-12). Thereafter, the association between sarcopenia and cytokines was analyzed using regression models.

Results: Low muscle strength and low-speed gait were negatively associated with IL-12 [ $\beta$ : -8.96 (95\% CI: $-14.12,-3.79)$ and -7.16 (95\% CI: $-12.54,-1.78)$, respectively]. Participants with more sarcopenia components and more severe sarcopenia had lower IL12 ( $\mathrm{P}$ for trend $<0.001$ ). Conversely, more amount of sarcopenia components were associated with increased TGF- $\beta$ ( $\mathrm{P}$ for trend $<0.05$ ). A definite diagnosis of sarcopenia was associated with decreased IL-12 and increased TGF- $\beta$ with $\beta$ of -8.96 (95\% CI: -14.12 , -3.79 ) and 147.75 (95\% CI: 36.27, 259.23). Furthermore, increased IL-12 levels were significantly associated with reduced occurrence of sarcopenia with and odd ratio (OR) of 0.36 (95\% CI: 0.15-0.834).

Conclusion: Our findings on the relationship between cytokines and age-related muscle loss showed that IL-12 may be an early diagnosis indicator for sarcopenia in the elderly population. Keywords: gender, sarcopenia, IL-12, TGF- $\beta$, macrophage

\section{Introduction}

Skeletal muscle aging, characterized by muscle mass loss, fibrosis, fat infiltration and other tissue modifications that causes a reduction in the force generated, has been associated with multiple adverse health outcomes, including loss of mobility, frailty, and sarcopenia. ${ }^{1-3}$ Age-related muscle loss is caused by potential mechanisms of imbalance between damage and repair processes at the molecular and myocellular levels. ${ }^{4-6}$ Inflammation is an important contributor to the pathology of diseases implicated in age-related skeletal muscle dysfunction. ${ }^{7}$ According to numerous studies, higher systemic concentrations of cytokines, ${ }^{8}$ greater basal cytokine production by peripheral blood mononuclear cells in vitro, ${ }^{9}$ and prolonged inflammatory reactions to infectious challenge in vivo ${ }^{10,11}$ are associated with ageing process. 
Macrophage polarization is a process by which macrophages adopt different functional programs in response to environmental signals, such as cytokine milieu, microbial ligands, and immune complexes. ${ }^{12}$ Mounting evidence has proposed that macrophage polarization promotes diverse physiological processes within the skeletal muscle, including myogenic differentiation, fibrosis, and revascularization. ${ }^{13-15}$ Emerging findings have suggested that M1 macrophages decline with aging, although M2 macrophages are predominant in human skeletal muscle. ${ }^{16}$ According to their specific functions, macrophages can be generally categorized into M1 and M2 subtypes, which produce pro-inflammatory and antiinflammatory cytokines, respectively. ${ }^{17} \mathrm{M} 1$ macrophages secrete tumor necrosis factor, interleukin-1 (IL-1), IL-10, IL-12, IL-23 and IL-6, whereas M2 macrophages secrete tumor growth factor- $\beta$ (TGF- $\beta$ ) and IL-1 receptor antagonist. In the present study, we analyzed the association between M1 and M2 macrophage activity in muscle tissues and adult age by a gene expression database and evaluated which cytokine was significantly associated with a specific macrophage. This cross-sectional study aimed to investigate the relationships between different cytokines and sarcopenia in an aging population in Taiwan.

\section{Methods}

\section{Study Design and Participant Recruitment}

In total, 120 elderly participants aged 65 years and older who had undergone health checkups at Tri-Service General Hospital (TSGH), Taiwan, from 2015 to 2019 were included in this cross-sectional study and the flow chart is shown in Figure 1. Questionnaires were used to obtain demographic information and muscle mass, muscle strength, and physical performance measurements, as well as laboratory examinations were performed. Thereafter, participants were categorized into four groups based on the number of sarcopenia components (component $0=36$; component $1=36$; component $2=32$; component $3=16$ ). TGF- $\beta$ and IL- 12 were then examined to explore their potential relationship with

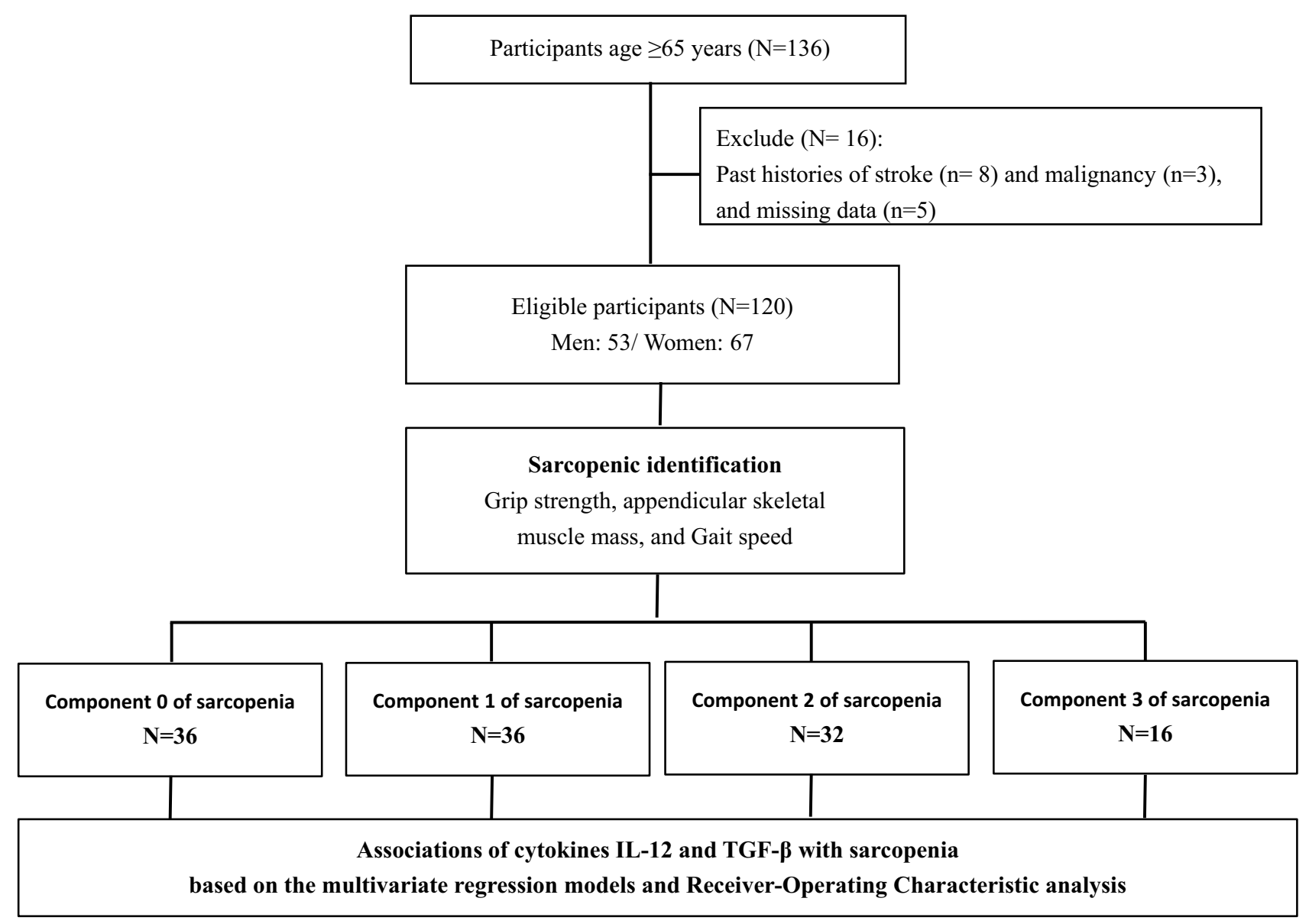

Figure I Flow chart of participants. 
sarcopenia components. All analytic procedures were conducted in accordance with the TSGH guidelines. Our study was approved by the Institutional Review Board of TSGH, Taiwan, and participants provided informed consent prior to enrollment. The study was conducted in accordance with the Declaration of Helsinki.

\section{Analysis of Macrophage and Cytokine Datasets from Gene Expression Omnibus Database}

Recent reviews evaluating immune cell populations and muscle homeostasis have shown that macrophages and their secreted cytokines were strongly correlated with muscle aging. ${ }^{18}$ To preliminarily assess the association between M1 and M2 macrophage activity in muscle tissues and adult age, GSE154846, a database of age-related gene expressions in adult skeletal muscle, was used (https://cordis.europa.eu/project/id/277936/reporting).

Approximately 500 samples of skeletal muscle from adults of different ages from GSE154846 was scored using Gene Set Variation Analysis (GSVA) scoring strategy based on published molecular signatures generated from M1 or M2 related differentially expressed genes. ${ }^{19}$ The young or middle-aged adult population had significantly higher GSVA scores representing M1 in their muscle tissue than the older population. In contrast, as expected, the GSVA scores for M2 increased significantly with age (Figure 2).
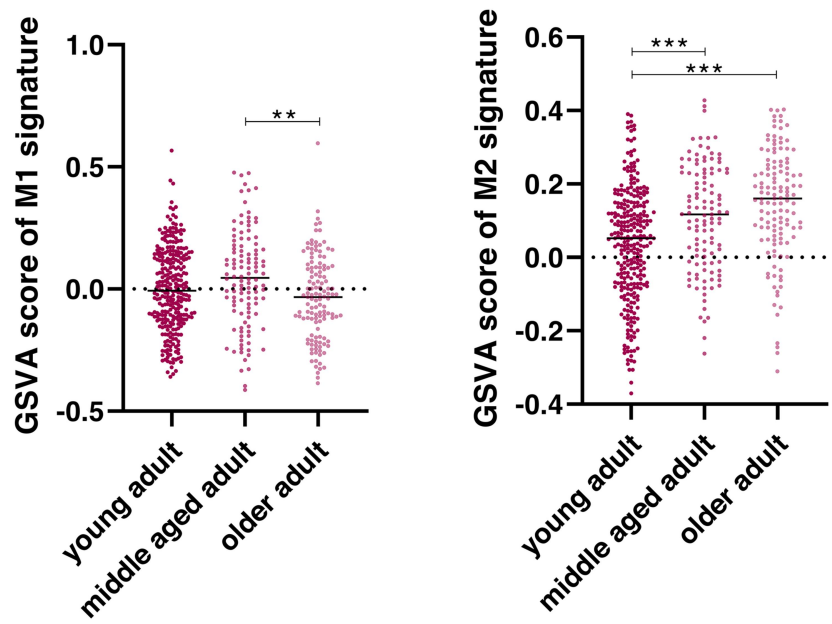

Figure 2 Gene set variation analysis scoring of $\mathrm{MI}$ and $\mathrm{M} 2$ signatures $\left({ }^{*} \mathrm{p}=0.005\right.$ $* * * p<0.001)$

\section{Sarcopenia Diagnosis}

The revised operational definition proposed by the 2019 European Working Group on Sarcopenia in Older People $(\text { EWGSOP2 })^{20}$ identifies low muscle strength as the primary criterion for sarcopenia. Specifically, the aforementioned guideline demonstrates how to diagnose and evaluate severity in clinical practice. Accordingly, sarcopenia is suspected when low muscle strength is observed and is subsequently diagnosed when low skeletal muscle mass is evident. Meanwhile, severe sarcopenia is considered when low physical performance and the two other criteria are observed. During the health checkups, muscle strength was measured through hand grip strength using an analog isometric dynamometer (North Coast Hydraulic Hand Dynamometer, North Coast Medical Inc., Morgan Hill, CA), while muscle quality or quantity was estimated through the skeletal mass index (SMI) by dividing the limb skeletal muscle mass $(\mathrm{kg})$ by the square of the height $\left(\mathrm{m}^{2}\right)$ using a BIA (InBody720, Biospace, Inc., Cerritos, CA, USA). Physical performance was measured using the 6-m walking distance. Based on the recommendations provided by EWGSOP2, the cutoff points for hand grip strength were 27.0 and $16 \mathrm{~kg}$ for men and women, respectively. ${ }^{21}$ Those for SMI were 7.0 and $6.0 \mathrm{~kg} / \mathrm{m} 2$ for men and women, respectively, ${ }^{22}$ and those for gait speed were $0.8 \mathrm{~m} / \mathrm{s}$ for both men and women. ${ }^{23}$

\section{Cytokines}

To determine the underlying pathophysiological mechanisms, the following cytokines were analyzed based on the standard procedures indicated in the manufacturer's instructions. TGF- $\beta$ (Cedar Valley Drive, Westlake Village, CA, USA) and IL-12 (Cedar Valley Drive, Westlake Village, CA, USA) were assayed using a competitive enzyme-linked immunoassay. The lower detection limits for TGF- $\beta$ and IL-12 were 15.6 and 7.8 $\mathrm{pg} / \mathrm{mL}$, respectively. The coefficients of variation for the four assays ranged from $5 \%$ to $10 \%$ during the sample analysis period.

\section{Study Variables}

Demographic data, such as age, gender, and smoking history, were obtained from participants' self-reported questionnaires. Information on comorbidities, such as hypertension, diabetes mellitus, myocardial infarction, angina, coronary artery disease, chronic obstructive 
pulmonary disease, and arthritis, was also collected from the participants.

\section{Statistical Analysis}

The Statistical Package for the Social Sciences, version 22.0 (SPSS Inc., Chicago, IL, USA), for Windows was used for all statistical analyses. All estimates were performed based on the sample weights to represent the total population of Taiwan and were adjusted for the complex sample design of the survey. Significant differences among sarcopenia component groups were determined using the Pearson's chi-square test. A two-sided $p$ value of $<0.05$ was considered statistically significant. Associations between cytokines and sarcopenia, as well as components thereof, were analyzed using a linear regression model. Moreover, relationships between cytokines and odds ratios (ORs) for sarcopenia were analyzed using a logistic regression model. Thereafter, to adjust for age, sex, smoking history, and number of comorbidities, multivariate analysis was conducted. Receiving operating characteristic (ROC) curve analysis was used to calculate the optimal cutoff points for cytokines as well as the area under the ROC (AUROC) and the corresponding 95\% confidence intervals $(\mathrm{CI})$ to determine the associations between cytokines and sarcopenia. Trend tests were assessed by treating the number of sarcopenia components as a continuous variable to observe the associations across an increasing number of sarcopenia components and cytokines.

\section{Results}

\section{Association Between Cytokines and Macrophage Polarization in the GEO Database}

M1 or M2 macrophages secrete distinct cytokines that affect the growth and regeneration of skeletal muscle, respectively. To evaluate which cytokine was significantly associated with the GSVA score of a specific macrophage, correlations between the aforementioned cytokine-related pathways after GSVA scoring via the NIH PID pathway and BIOCARTA databases were further analyzed.

Figure 3 shows that the GSVA score of the IL12 pathway significantly differed among adult populations of different ages and was significantly positively correlated with M1 macrophage score $(\mathrm{p}<0.001 ; \mathrm{r}=0.319)$. In contrast, the GSVA score of the TGFB pathway was significantly negatively correlated with the M1 macrophage GSVA score $(\mathrm{p}=0.010 ; \mathrm{r}=-0.111)$. Other cytokines were either not significantly correlated with M1/M2 GSVA scores across age groups or were not significantly correlated with M1/ M2 GSVA scores.

\section{Study Population Characteristics}

All participants were categorized into four groups based on the number of sarcopenia components, and Table 1 summarizes the demographic characteristics of participants. The mean age was significant different in each group with 72.29 \pm 5.46, $72.36 \pm 6.71,75.98 \pm 8.77,82.44 \pm 8.74$ years, respectively. Participants with more sarcopenia components had significantly lower grip strength, physical performance, and skeletal muscle mass $(\mathrm{p}<0.001)$.

\section{Association Between Sarcopenia and Cytokines}

Table 2 presents the associations between cytokines and sarcopenia components, number of sarcopenia components, and definite diagnosis of sarcopenia. Accordingly, the univariate model showed that low muscle strength, muscle mass, and speed gait were associated with decreased IL-12 levels with $\beta$ of -6.69 (95\% CI: -11.25 , $-2.13),-4.68$ (95\% CI: $-9.25,-0.10)$, and -6.68 (95\% CI: $-11.74,-1.82)$, respectively. After adjusting for variables including age, sex, smoking history, and number of comorbidities, low muscle strength and low-speed gait remained significantly associated with decreased IL-12 levels with $\beta$ of -8.96 (95\% CI: $-14.12,-3.79 ; \mathrm{p}<0.001)$ and -7.16 (95\% CI: $-12.54,-1.78 ; \mathrm{p}=0.010)$. Participants with more sarcopenia components had significantly lower IL12 with $\beta$ of -4.55 ( $\mathrm{P}$ for trend $<0.001$ ). Confirmed and severe sarcopenia were associated with decreased IL-12 with $\beta$ of -7.51 (95\% CI: $-13.94,-1.07 ; \mathrm{p}=0.023)$ and $-14.03(95 \%$ CI: $-21.36,-6.69 ; \mathrm{p}<0.001)$ after fully adjusting age, sex, smoking history, and number of comorbidities. Conversely, low muscle strength was associated with increased TGF- $\beta$ levels with $\beta$ of 147.75 (95\% CI: 36.27, 259.23; $\mathrm{p}=0.010$ ). More sarcopenia components were significantly associated with higher TGF- $\beta$ with $\beta$ of 52.17 ( $\mathrm{P}$ for trend $<0.05$ ). Confirmed sarcopenia was associated with increased TGF- $\beta$ with $\beta$ of 190.10 (95\% CI: 50.08, 330.13; $\mathrm{p}=0.008)$. Moreover, a definite diagnosis of sarcopenia, including confirmed and severe subtypes, was significantly associated with decreased IL-12 and increased TGF- $\beta$ with $\beta$ of -8.96 (95\% CI: -14.12 , $-3.79)$ and 147.75 (95\% CI: 36.27, 259.23) after full adjustment. 
M1:IL12
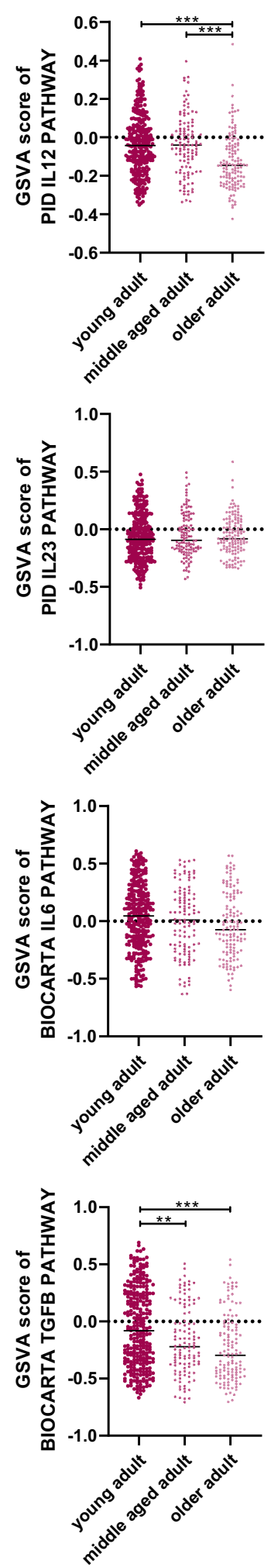

M1:IL23

M1:IL6
M2:IL12
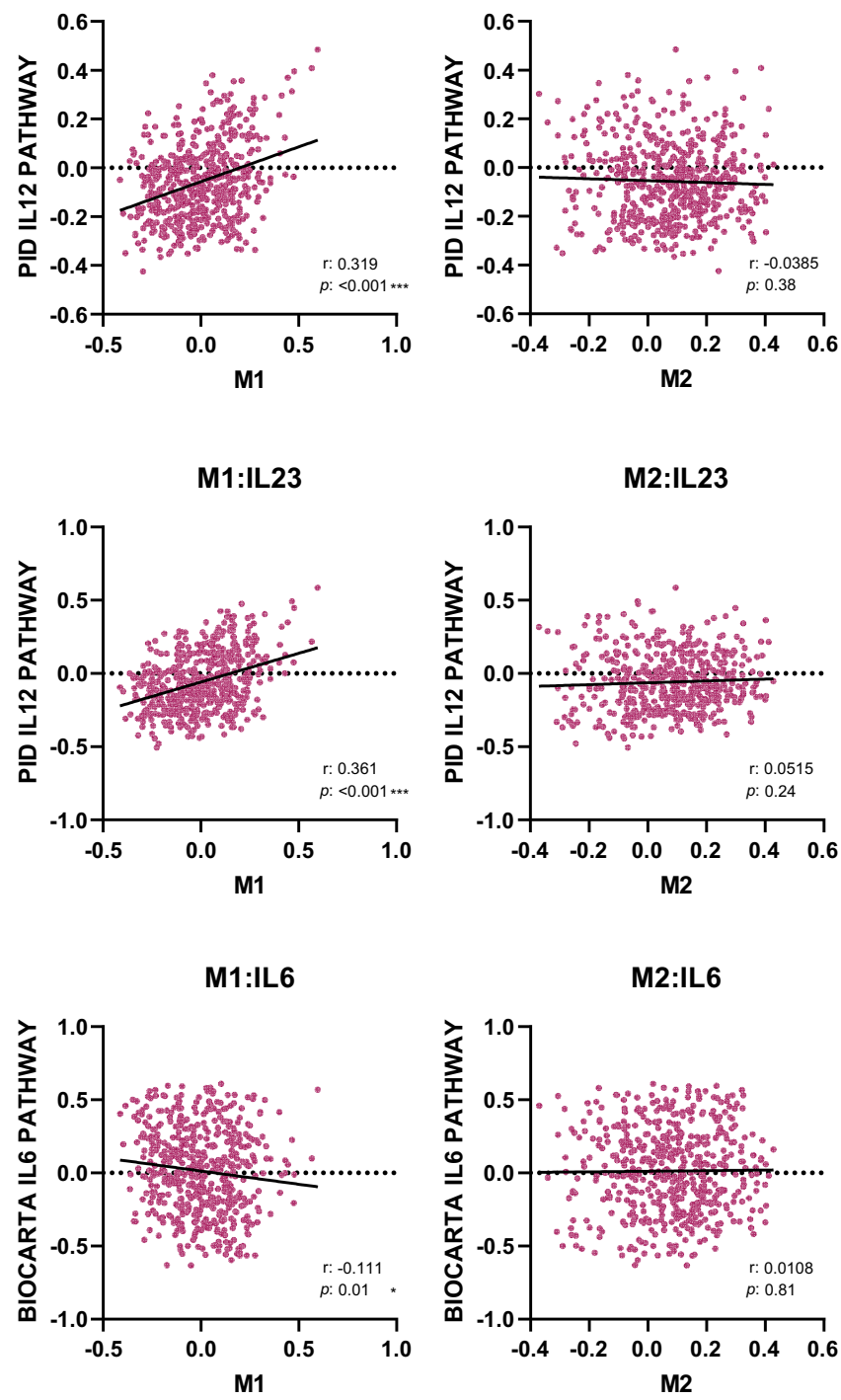

M2:IL6

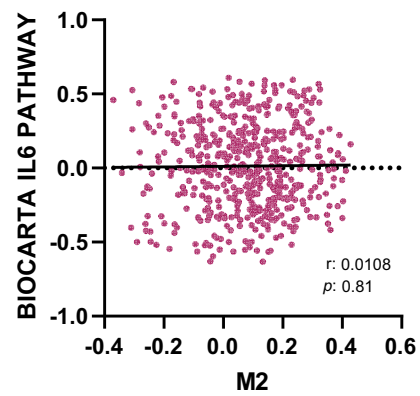

M1:TGFB
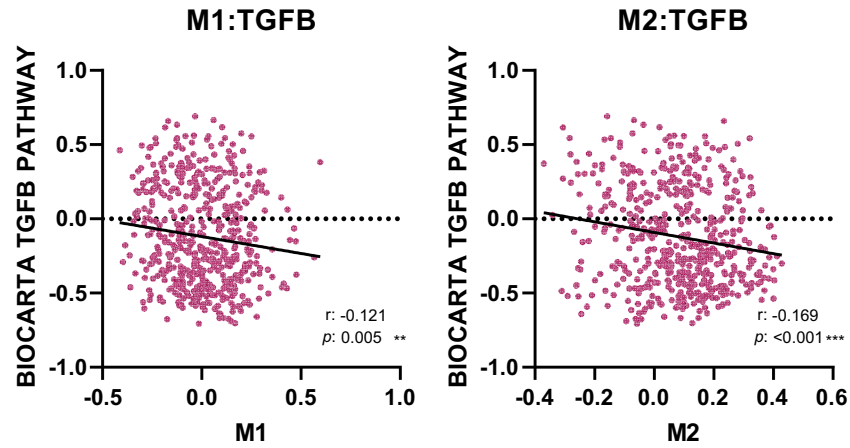

Figure 3 Associations among different cytokines expressions, MI, and M2 signatures $(* * \mathrm{p}=0.005 / * * * p<0.00 \mathrm{I})$. 
Table I Characteristics of Study Population

\begin{tabular}{|c|c|c|c|c|c|}
\hline \multirow{2}{*}{ Variable } & \multicolumn{5}{|c|}{ Components of Sarcopenia } \\
\hline & $\mathbf{0}$ & $\mathbf{I}$ & 2 & 3 & $P$ value \\
\hline \multicolumn{6}{|l|}{ Continuous variable (SD) } \\
\hline Age (years) & $72.29(5.46)$ & $72.36(6.7 I)$ & $75.98(8.77)$ & $82.44(8.74)$ & $<0.001$ \\
\hline Grip strength (kg) & $30.08(8.69)$ & $25.30(7.86)$ & $19.25(8.09)$ & $15.62(5.90)$ & $<0.001$ \\
\hline $\mathrm{SMI}\left(\mathrm{kg} / \mathrm{m}^{2}\right)$ & $7.11(0.89)$ & $6.08(0.99)$ & $5.76(0.8 I)$ & $5.88(0.67)$ & $<0.001$ \\
\hline Gait speed $(\mathrm{m} / \mathrm{s})$ & $1.20(0.18)$ & $1.19(0.31)$ & $0.88(0.26)$ & $0.59(0.16)$ & $<0.001$ \\
\hline Comorbidity No. & $0.78(0.96)$ & $0.89(1.19)$ & $1.03(1.15)$ & $1.06(1.06)$ & 0.746 \\
\hline IL-I2 (pg/mL) & $46.08(12.94)$ & $45.05(14.28)$ & $40.11(7.66)$ & 33.71 (10.59) & 0.003 \\
\hline TGF- $\beta$ (pg/mL) & $139.34(145.36)$ & |49.2| (173.09) & $27 \mid .32(361.31)$ & $289.69(381.05)$ & 0.072 \\
\hline \multicolumn{6}{|l|}{ Categorical variable (\%) } \\
\hline Gender (male) & $18(50.0)$ & $20(55.6)$ & $22(68.8)$ & $7(43.8)$ & 0.674 \\
\hline Smoking & $11(31.4)$ & $9(25)$ & $4(12.5)$ & $2(12.5)$ & 0.043 \\
\hline HTN & $15(41.7)$ & $10(27.8)$ & $13(40.6)$ & $10(62.5)$ & 0.219 \\
\hline DM & $6(16.7)$ & $6(16.7)$ & $5(15.6)$ & I (6.3) & 0.428 \\
\hline MI & $0(0.0)$ & I (2.8) & $0(0.0)$ & $0(0.0)$ & 0.821 \\
\hline Angina & $0(0.0)$ & $0(0.0)$ & $0(0.0)$ & $0(0.0)$ & - \\
\hline CAD & $3(8.3)$ & $3(8.3)$ & $3(9.4)$ & $2(12.5)$ & 0.661 \\
\hline COPD & $2(5.6)$ & $2(5.6)$ & $2(6.3)$ & $0(0.0)$ & 0.570 \\
\hline Arthritis & $2(5.6)$ & $10(27.8)$ & $10(31.3)$ & $4(25.0)$ & 0.031 \\
\hline
\end{tabular}

Abbreviations: SMI, skeletal muscle mass index; S\&S time, stand up and sit down time; HTN, hypertension; DM, diabetes mellitus; MI, myocardial infarction; CAD, coronary artery disease; COPD, chronic obstructive pulmonary disease.

\section{Association Between Cytokines and Occurrence of Sarcopenia}

To determine the ability of cytokines to predict the occurrence of sarcopenia, the optimal cutoff points for IL-12 and TGF- $\beta$ were calculated using ROC analysis (Figure 4). Accordingly, IL-12 had an AUROC of 0.64 (95\% CI: $0.53-0.74 ; \mathrm{p}<0.001)$ with an optimal cutoff point of 41.80 (sensitivity: 53\%; specificity: 73\%). Meanwhile, TGF- $\beta$ had an AUROC of 0.60 (95\% CI: $0.49-0.72$; $\mathrm{p}<0.001$ ) with an optimal cutoff point of 92.09 (sensitivity: 66\%; specificity: 56\%) (Table 3).

Figure 5 shows the relationships between the occurrence of sarcopenia and continuous cytokine levels and their cutoff points. IL-12 and age were associated with the occurrence of sarcopenia with OR of 0.969 (95\% CI: $0.939-1.000 ; \mathrm{p}=0.050)$ and 1.061 (95\% CI: $1.003-1.121$; $\mathrm{p}=0.037$ ) in the unadjusted model. After full adjustment, IL-12 remained significantly associated with the occurrence of sarcopenia with OR of 0.969 (95\% CI: 0.938-$1.000 ; \mathrm{p}=0.050$ ). However, no significant association was noted between TGF- $\beta$ and sarcopenia. Regarding cytokine cutoff points, IL-12 was inversely associated with the occurrence of sarcopenia with OR of 0.356 (95\% CI:
0.157-0.807; $\mathrm{p}=0.018$ ) (Figure 5), while TGF- $\beta$ was positively associated with the occurrence of sarcopenia with OR of 2.443 ( $95 \%$ CI: $1.080-5.527$; $\mathrm{p}=0.032$ ) in the unadjusted model. After full adjustment for variables, IL-12 remained significantly associated with the occurrence of sarcopenia with OR of 0.356 (95\% CI: $0.151-0.838$; $\mathrm{p}=0.018$ ).

\section{Discussion}

The present study found that the cytokine levels might affect the number of sarcopenia components and sarcopenia severity. Accordingly, our results showed that, among elderly participants, more sarcopenia components were associated with decreased IL-12 and increased TGF- $\beta$ levels. We speculate that these cytokines might have been partly released by muscle differentiation during macrophage polarization into M1 and M2 subtypes, which are involved in pro-inflammatory and anti-inflammatory activities, respectively. To the best of our knowledge, the present study is the first to explore the relationships between cytokines and sarcopenia in a Taiwanese elderly population.

TGF- $\beta$ has been proposed to induce skeletal muscle atrophy and fibrosis by regulating the activity of skeletal 


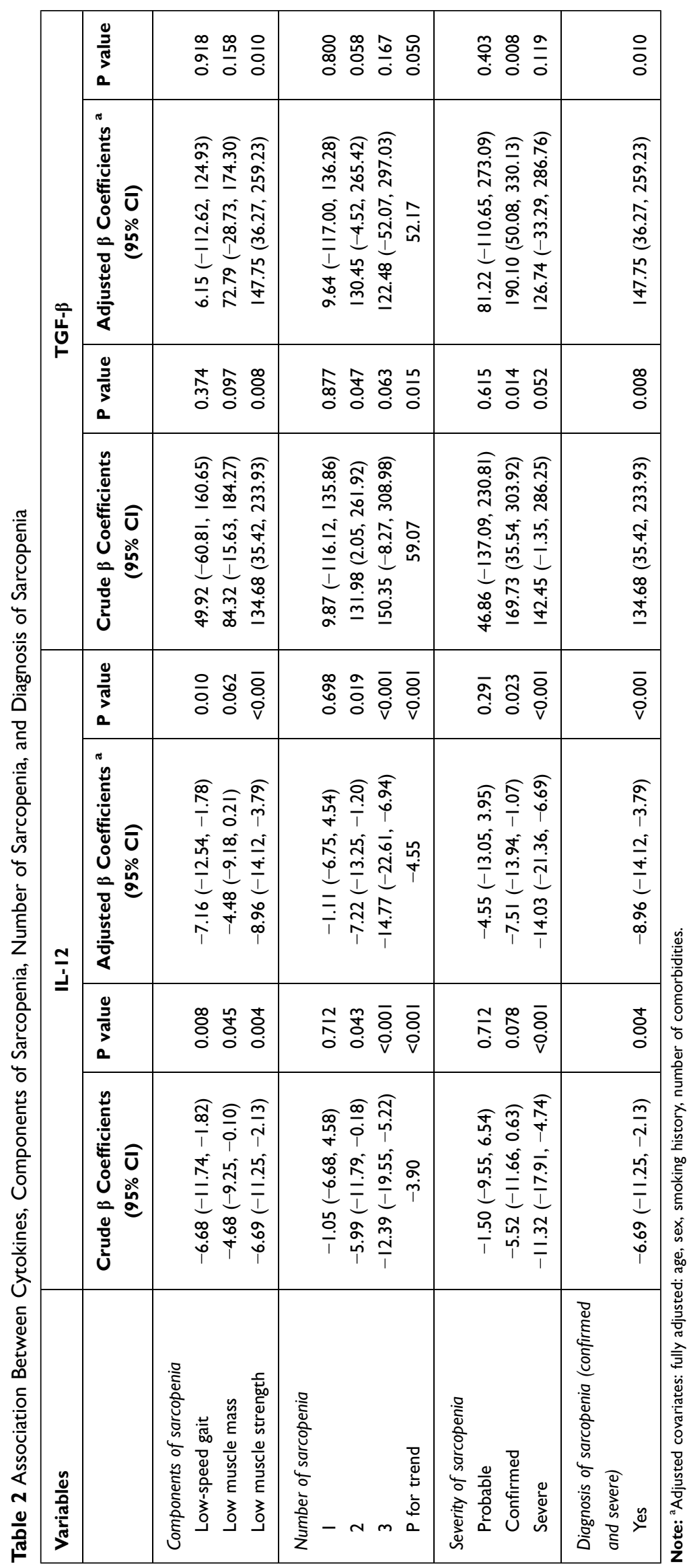




\section{A}

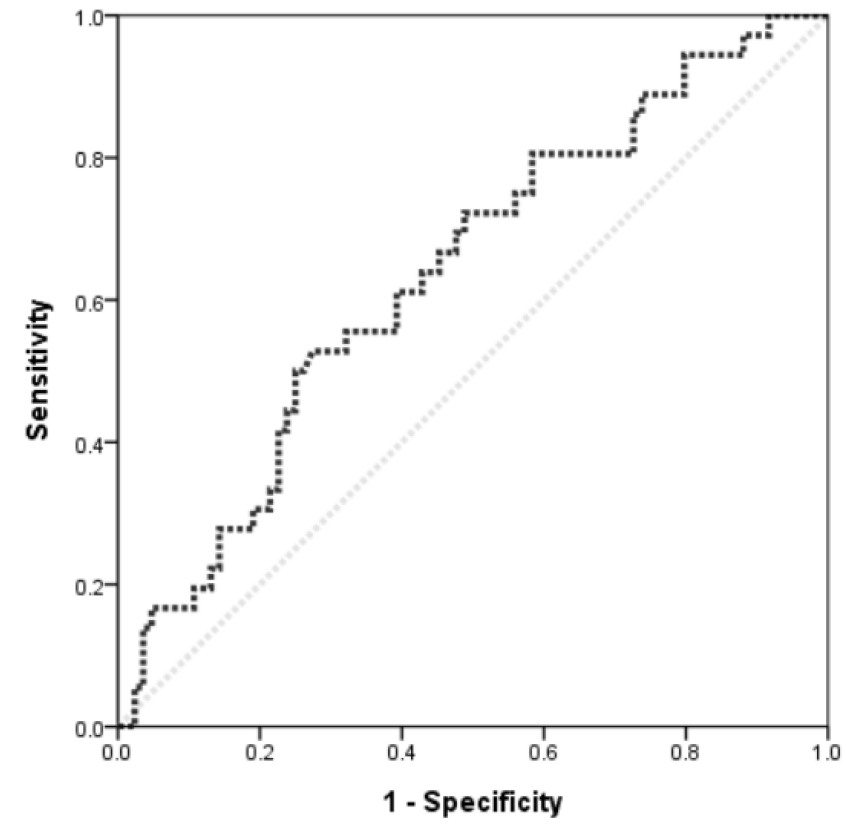

B

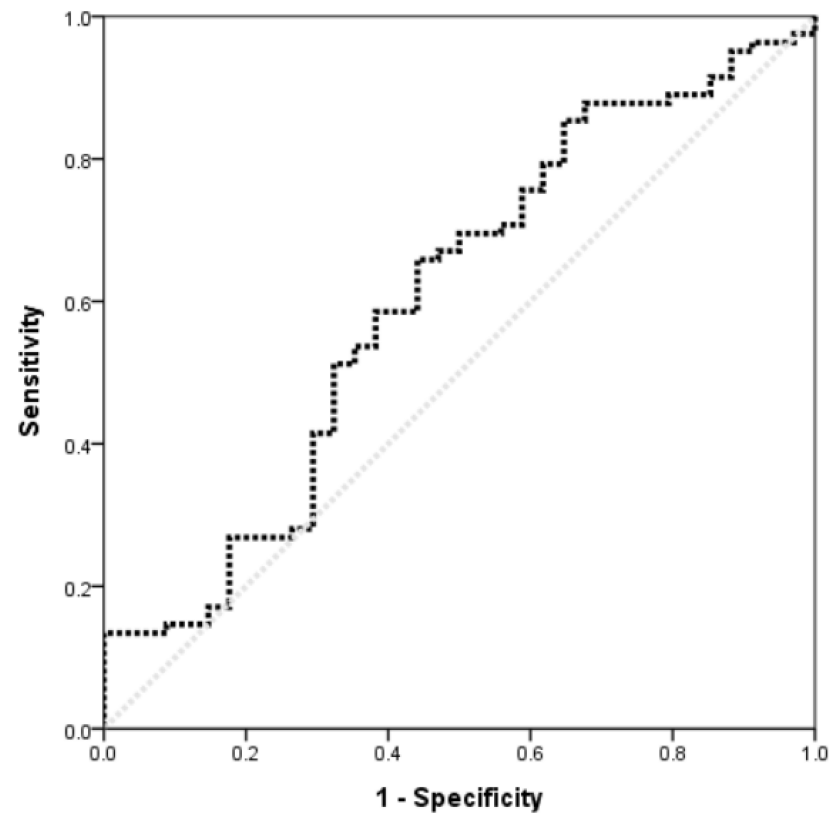

Figure 4 Receiver operating characteristic curve analysis for optimal cutoff points of IL-I2 (A) and TGF- $\beta$ (B).

muscle satellite cell. ${ }^{24}$ Moreover, TGF- $\beta$ has been suggested to lower muscle regeneration by inhibiting muscle cell differentiation and proliferation via the Smad-3 signaling system, which has been shown to prevent positive regulators of muscle cell differentiation, including MyoD and myogenin. ${ }^{25}$ Excessive TGF- $\beta$ production has been considered the main cause of progressive tissue fibrosis and scar formation, leading to muscle loss and impaired muscle function. ${ }^{18}$ Moreover, studies have suggested that TGF- $\beta$ plays a role in muscle impairment and fibrosis accompanying the aging process. ${ }^{18}$ Our findings are consistent with those presented in previous studies showing that sarcopenia is significantly associated with increased TGF- $\beta$ levels through various processes, such as muscle damage, regeneration, and fibrosis. ${ }^{26}$

Table 3 Optimal Cutoff Points of Cytokines

\begin{tabular}{|l|c|c|}
\hline & IL-I 2 & TGF- $\boldsymbol{\beta}$ \\
\hline AUC $(95 \% \mathrm{Cl})$ & $0.64(0.53-0.74)$ & $0.60(0.49-0.72)$ \\
\hline Sensitivity & $53 \%$ & $66 \%$ \\
\hline Specificity & $73 \%$ & $56 \%$ \\
\hline P-value & 0.018 & 0.084 \\
\hline Cutoff points & 41.80 & 92.09 \\
\hline
\end{tabular}

IL-12 is a pro-inflammatory cytokine that promotes $\mathrm{T}$ cell proliferation and cytotoxic activity. ${ }^{27}$ Unfortunately, limited studies have been available regarding the role of IL-12 in myogenic differentiation. Romanazzo et al demonstrated that IL-12 overexpression in $\mathrm{C} 2 \mathrm{C} 12$ mouse skeletal muscle cells enhanced the myogenic differentiation process. ${ }^{28}$ IL-12 has been a wellknown risk factor for Alzheimer's disease and cognitive aging. ${ }^{29,30}$ A population-based study of Taiwanese elderly participants suggested that IL-12-associated genes caused the etiological changes observed during cognitive aging. ${ }^{31}$ Previous studies have also proposed a connection between IL-12 and physical activity. Accordingly, endurance exercise has been associated with the induction of Th2 cytokines, including IL-4 and IL-12, ${ }^{32}$ while Suzuki et al demonstrated that IL-12 concentration significantly increased after maximal exercise test. ${ }^{33}$ Moreover, a retrospective study of 16 men who participated in a running program showed that intense exercise enhances IL-12 levels in peripheral blood mononuclear cells. ${ }^{34}$ The present study found that lower IL-12 levels were associated with a higher occurrence of sarcopenia. Given the aforementioned findings, patients with increased IL-12 levels may have better muscle strength and physical performance, as well as lower incidences of sarcopenia. Further experiments will be necessary to clarify the 


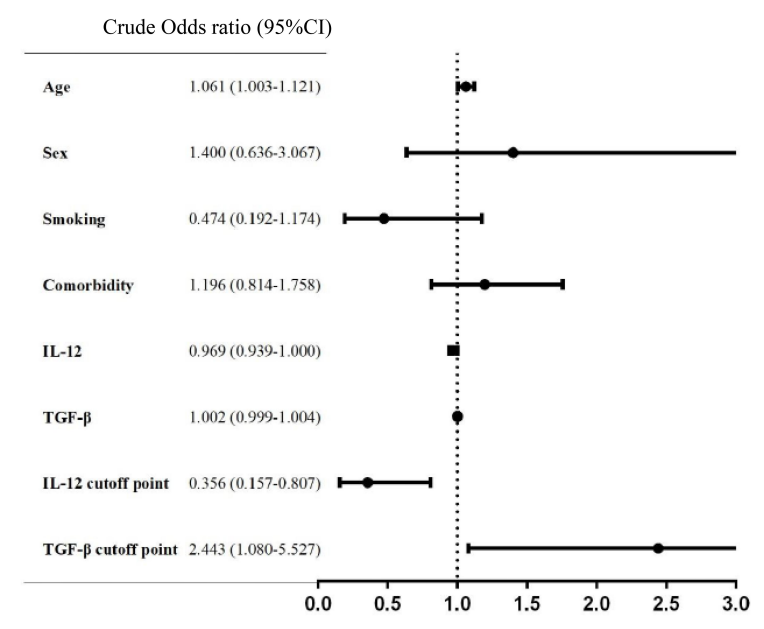

Figure 5 Association between cytokines and the occurrence of sarcopenia.

function of IL-12 in human skeletal muscle and the molecular mechanisms underlying its action.

Cui et al have reported the role of macrophage polarization in skeletal muscle metabolism associated with aging, which involves distinct macrophage function. ${ }^{16}$ Impaired macrophage activity during aging has been suggested to promote deterioration in satellite cell function and muscle regeneration. ${ }^{35}$ One study observed that the resting muscles of aged mice had increased M2 macrophages, accompanied by increased skeletal muscle fibrosis. ${ }^{36}$ Meanwhile, a human study showed that older patients with type 2 diabetes mellitus had higher gene expression of M2 macrophages compared to the younger patients, suggesting an increase in the proinflammatory process with age. ${ }^{37}$ Moratal et al demonstrated that M2 macrophages induced adipogenesis through fibroadipogenic progenitors. ${ }^{38}$ By promoting fibrosis and fat infiltration, which are major features of skeletal muscle aging, macrophage polarization, especially M2 macrophages, may play an important role in the altered skeletal muscle function, and sarcopenia.

Some limitations of the present study are worth noting. First, the cross-sectional design of this study prevented the identification of causal relationships between cytokines and sarcopenia. Therefore, longitudinal studies are needed to explore whether cytokines can be utilized for the early diagnosis and evaluation of therapeutic interventions in sarcopenia. Second, flow cytometry, which measures specific protein expressions, has been the standard method for identifying macrophages. However, given that only a few macrophages were detected in the study samples, cytokines were obtained from participants' blood samples to indicate macrophage

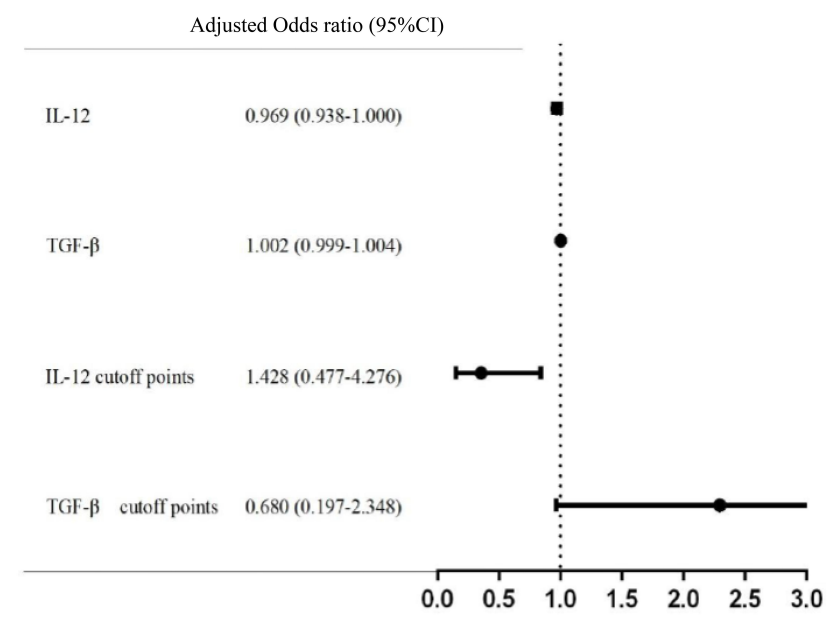

activation. Next, considering that cytokines are potent mediators across various inflammatory states and age-related diseases, their measurements might have been affected by physiological and pathological changes. Therefore, macrophage polarization can only be an indirect predictor for evaluating muscle differentiation and sarcopenia. Fourth, the information of baseline physical activity levels, which is the potential confounding factor in the association between IL-12 and sarcopenia, was unavailable in this study. Finally, the sensitivity and specificity values are relatively low in the present study. The sensitivity and specificity of a quantitative test are dependent on the cutoff point above or below which the test is positive. If the cutoff point is raised, there are fewer false positives but more false negatives than the test is highly specific but not very sensitive.

The current study found that the number of sarcopenia components and sarcopenia severity were associated with plasma cytokines levels among older individuals. Furthermore, IL-12 was found to be associated with the occurrence of sarcopenia, suggesting its potential as a muscle quality indicator for predicting outcomes of age-associated muscle loss. The combination of clinical practice and biomarker assessment in evaluating muscle quality may be essential for the early diagnosis of sarcopenia. Moreover, determining whether IL-12 can become a novel biological target for preventive and therapeutic interventions will be interesting. Nevertheless, the potential mechanisms through which macrophage function affects aging skeletal muscle remain unclear. Further studies are required to characterize the function of these cytokines and provide a basis for the better modulation of their signaling in therapeutic approaches. 


\section{Data Sharing Statement}

The datasets generated and analyses performed during the current study are not publicly available due to the consent requirement of participants, but sex and age decadestratified descriptive data are available from the corresponding author on reasonable request.

\section{Ethics Approval and Consent to Participate}

All procedures were conducted in accordance with the guidelines of TSGH. We have obtained patient permission before enrollment by asking them to complete a written informed consent, and approval for the study was granted by the Institutional Review Board of TSGH, Taiwan.

\section{Consent for Publication}

Not applicable.

\section{Author Contributions}

All authors made a significant contribution to the work reported, whether that is in the conception, study design, execution, acquisition of data, analysis and interpretation, or in all these areas; took part in drafting, revising or critically reviewing the article; gave final approval of the version to be published; have agreed on the journal to which the article has been submitted; and agree to be accountable for all aspects of the work.

\section{Funding}

This study was supported by the Ministry of Science and Technology (MOST 108-2314-B-016 -032), Taiwan, ROC.

\section{Disclosure}

The authors declared that they had no competing interests.

\section{References}

1. Janssen I. Influence of sarcopenia on the development of physical disability: the Cardiovascular Health Study. $J$ Am Geriatr Soc. 2006;54:56-62. doi:10.1111/j.1532-5415.2005.00540.x

2. Ferrucci L. The origins of age-related proinflammatory state. Blood. 2005;105:2294-2299. doi:10.1182/blood-2004-07-2599

3. Tieland M, Trouwborst I, Clark BC. Skeletal muscle performance and ageing. J Cachexia Sarcopenia Muscle. 2018;9(1):3-19. doi:10.1002/ jcsm. 12238

4. Hepple RT, Rice CL. Innervation and neuromuscular control in ageing skeletal muscle. J Physiol. 2016;594(8):1965-1978. doi:10.1113/ jp270561
5. Saini J, McPhee JS, Al-Dabbagh S, Stewart CE, Al-Shanti N. Regenerative function of immune system: modulation of muscle stem cells. Ageing Res Rev. 2016;27(67):67-76. doi:10.1016/j. arr.2016.03.006

6. Gonzalez-Freire M, de Cabo R, Studenski SA, Ferrucci L. The neuromuscular junction: aging at the crossroad between nerves and muscle. Front Aging Neurosci. 2014;6(208). doi:10.3389/ fnagi.2014.00208

7. Dalle S, Rossmeislova L, Koppo K. The Role of Inflammation in Age-Related Sarcopenia. Front Physiol. 2017;8(1045). doi:10.3389/ fphys.2017.01045

8. Wikby A. The immune risk phenotype is associated with IL-6 in the terminal decline stage: findings from the Swedish NONA immune longitudinal study of very late life functioning. Mech Ageing Dev. 2006;127(8):695-704. doi:10.1016/j.mad.2006.04.003

9. Roubenoff R. Monocyte cytokine production in an elderly population: effect of age and inflammation. J Gerontol a Biol Sci Med Sci. 1998;53:M20-M26. doi:10.1093/gerona/53a.1.m20

10. Krabbe KS. Ageing is associated with a prolonged fever response in human endotoxemia. Clin Diagn Lab Immunol. 2001;8:333-338. doi:10.1128/cdli.8.2.333-338.2001

11. Bruunsgaard H, Skinhoj P, Qvist J, Pedersen BK. Elderly humans show prolonged in vivo inflammatory activity during pneumococcal infections. J Infect Dis. 1999;180:551-554. doi:10.1086/314873

12. Wynn TA, Chawla A, Pollard JW. Macrophage biology in development, homeostasis and disease. Nature. 2013;496:445-455. doi:10.1038/nature12034

13. Tidball JG. Mechanisms of muscle injury, repair, and regeneration. Compr Physiol. 2011;1:2029-2062. doi:10.1002/cphy.c100092

14. Dufresne SS, Frenette J, Dumont NA. [Inflammation and muscle regeneration, a double-edged sword]. Med Sci (Paris). 2016;32:591-597. doi:10.1051/medsci/20163206022

15. Smith C, Kruger MJ, Smith RM, Myburgh KH. The inflammatory response to skeletal muscle injury: illuminating complexities. Sports Med. 2008;38:947-969. doi:10.2165/00007256-20083811000005

16. Cui CY. Skewed macrophage polarization in aging skeletal muscle. Aging Cell. 2019;18(e13032). doi:10.1111/acel.13032

17. Mills CD, Kincaid K, Alt JM, Heilman MJ, Hill AM. M-1/M-2 macrophages and the Th1/Th2 paradigm. J Immunol. 2000;164 (12):6166-6173. doi:10.4049/jimmunol.164.12.6166

18. Ismaeel A. Role of transforming growth factor- $\beta$ in skeletal muscle fibrosis: a review. Int J Mol Sci. 2019;20(2446):2446. doi:10.3390/ ijms20102446

19. Foroutan M. Single sample scoring of molecular phenotypes. $B M C$ Bioinform. 2018;19:404. doi:10.1186/s12859-018-2435-4

20. Cruz-Jentoft AJ. Sarcopenia: revised European consensus on definition and diagnosis. Age Ageing. 2019;48(16-31):601. doi:10.1093/ ageing/afy 169

21. Dodds RM. Grip strength across the life course: normative data from twelve British studies. PLoS One. 2014;9(:e113637. doi:10.1371/ journal.pone. 0113637

22. Gould H, Brennan SL, Kotowicz MA, Nicholson GC, Pasco JA. Total and appendicular lean mass reference ranges for Australian men and women: the Geelong osteoporosis study. Calcif Tissue Int. 2014;94:363-372. doi:10.1007/s00223-013-9830-7

23. Cruz-Jentoft AJ. Sarcopenia: European consensus on definition and diagnosis: report of the European working group on sarcopenia in older people. Age Ageing. 2010;39:412-423. doi:10.1093/ageing/ afq034

24. Mendias CL. Transforming growth factor-beta induces skeletal muscle atrophy and fibrosis through the induction of atrogin- 1 and scleraxis. Muscle Nerve. 2012;45(1):55-59. doi:10.1002/mus.22232

25. Carlson ME, Hsu M, Conboy IM. Imbalance between pSmad3 and Notch induces CDK inhibitors in old muscle stem cells. Nature. 2008;454(7203):528-532. doi:10.1038/nature07034 
26. Kim J, Lee J. Role of transforming growth factor- $\beta$ in muscle damage and regeneration: focused on eccentric muscle contraction. $J$ Exerc Rehabil. 2017;13(6):621-626. doi:10.12965/jer.1735072.536

27. Trinchieri G. Interleukin-12: a proinflammatory cytokine with immunoregulatory functions that bridge innate resistance and antigen-specific adaptive immunity. Annu Rev Immunol. 1995;13 (10):251-276. doi:10.1146/annurev.iy.13.040195.001343

28. Romanazzo S, Forte G, Morishima K, Taniguchi A. IL-12 involvement in myogenic differentiation of $\mathrm{C} 2 \mathrm{C} 12$ in vitro. Biomater Sci. 2015;3(3):469-479. doi:10.1039/c4bm00315b

29. Vom Berg J. Inhibition of IL-12/IL-23 signaling reduces Alzheimer's disease-like pathology and cognitive decline. Nat Med. 2012;18 (12):1812-1819. doi:10.1038/nm.2965

30. Tan MS. IL12/23 p40 inhibition ameliorates Alzheimer's diseaseassociated neuropathology and spatial memory in SAMP8 mice. J Alzheimers Dis. 2014;38:633-646. doi:10.3233/jad-131148

31. Lin E, Kuo PH, Liu YL, Yang AC, Tsai SJ. Association and interaction effects of interleukin-12 Related genes and physical activity on cognitive aging in old adults in the Taiwanese population. Front Neurol. 2019;10(1065). doi:10.3389/fneur.2019.01065

32. Suzuki K. Cytokine Response to Exercise and Its Modulation. Antioxidants (Basel). 2018;7(17). doi:10.3390/antiox7010017
33. Suzuki K. Exhaustive exercise and type-1/type-2 cytokine balance with special focus on interleukin-12 p40/p70. Exerc Immunol Rev. 2003;9(48):48-57.

34. Zamani A, Salehi I, Behzad M. Moderate exercise enhances the production of interferon- $\gamma$ and interleukin-12 in peripheral blood mononuclear cells. Immune Netw. 2017;17(3):186. doi:10.4110/ in.2017.17.3.186

35. Dort J, Fabre P, Molina T, Dumont NA. Macrophages are key regulators of stem cells during skeletal muscle regeneration and diseases. Stem Cells Int. 2019;4761427. doi:10.1155/2019/4761427

36. Wang Y, Wehling-Henricks M, Samengo G, Tidball JG. Increases of M2a macrophages and fibrosis in aging muscle are influenced by bone marrow aging and negatively regulated by muscle-derived nitric oxide. Aging Cell. 2015;14:678-688. doi:10.1111/acel.12350

37. Tam CS. Low macrophage accumulation in skeletal muscle of obese type 2 diabetics and elderly subjects. Obesity (Silver Spring). 2012;20 (7):1530-1533. doi:10.1038/oby.2012.24

38. Moratal C. IL-1 $\beta$ - and IL-4-polarized macrophages have opposite effects on adipogenesis of intramuscular fibro-adipogenic progenitors in humans. Sci Rep. 2018;8(17005). doi:10.1038/s41598-018-35429-w
Journal of Inflammation Research

\section{Publish your work in this journal}

The Journal of Inflammation Research is an international, peerreviewed open-access journal that welcomes laboratory and clinical findings on the molecular basis, cell biology and pharmacology of inflammation including original research, reviews, symposium reports, hypothesis formation and commentaries on: acute/chronic inflammation; mediators of inflammation; cellular processes; molecular

\section{Dovepress}

mechanisms; pharmacology and novel anti-inflammatory drugs; clinical conditions involving inflammation. The manuscript management system is completely online and includes a very quick and fair peerreview system. Visit http://www.dovepress.com/testimonials.php to read real quotes from published authors. 